\title{
Utilizando Agentes e Ontologia de Modelo de Aluno Aberto para Prover Adaptação de Conteúdos Construtivistas em Ambientes Virtuais de Aprendizagem
}

\author{
Vitor Bremgartner ${ }^{1,2}$, José Francisco M. Netto $^{1}$, Crediné Menezes ${ }^{3}$ \\ ${ }^{1}$ Instituto de Computação (IComp) - Universidade Federal do Amazonas (UFAM) \\ Av. Gen. Rodrigo Otávio, Coroado. Manaus - AM - Brasil \\ ${ }^{2}$ Instituto Federal do Amazonas (IFAM) - Campus Manaus Distrito Industrial \\ Av. Danilo Areosa, Distrito Industrial. Manaus - AM - Brasil \\ ${ }^{3}$ Faculdade de Educação - Universidade Federal do Rio Grande do Sul (UFRGS) \\ Av. Paulo Gama, Farroupilha. Porto Alegre - RS - Brasil \\ \{vitorbref, jnetto\}@icomp.ufam.edu.br, credine@gmail.com
}

\begin{abstract}
Typically, the existing resources in Virtual Learning Environments (VLEs), used in distance education courses and blended, are presented in the same way for all students. This may not be useful for effective learning of each learner. Faced with this problem, this paper presents a strategy that allows resources adaptation to students in VLEs based on a constructivist approach, using multi-agent system technology manipulating an open learner model ontology comprised of several learner characteristics, such as competencies, skills, equipment which the learner uses, performance on activities, frequency, and learning styles. The leaner model is dynamically changed during the course, through the interactions of the student with VLE. Moreover, this model is shown to the student, with the aim of the student to know their current status in the course. Results obtained in a Numerical Analysis discipline show the feasibility of the proposal.
\end{abstract}

Resumo. Normalmente, os recursos existentes em Ambientes Virtuais de Aprendizagem (AVAs), utilizados em cursos de educação a distância e em semipresenciais, são apresentados da mesma forma para todos os alunos. Isto pode não ser útil para a aprendizagem efetiva de cada aluno. Diante deste problema, este artigo apresenta uma estratégia que permite adaptação de recursos para alunos em AVAs com base em uma abordagem construtivista, utilizando a tecnologia de sistema multiagente que manipula uma ontologia de modelo de aluno aberto composto de várias características do aluno, tais como competências, habilidades, equipamento que o aluno utiliza, o desempenho nas atividades, frequência e estilos de aprendizagem. $O$ modelo de aluno é alterado dinamicamente durante o curso, através das interações do aluno com o AVA. Além disso, este modelo é apresentado para o aluno, com o objetivo de o aluno conhecer seu estado atual no curso. Resultados obtidos em uma disciplina de Cálculo Numérico mostram a viabilidade da proposta.

\section{Introdução}

A Educação a Distância (EaD) é uma modalidade amplamente utilizada nos processos de ensino-aprendizagem. Para apoiar os cursos de $\mathrm{EaD}$ ou semipresenciais existem os Ambientes Virtuais de Aprendizagem (AVAs), facilitando o processo de comunicação entre estudantes, professores, tutores e a comunidade, permitindo a todos participarem de um modo interativo com a disponibilidade de materiais didáticos. Além disso, existem AVAs que utilizam uma 
camada de inteligência, especialmente no que diz respeito à possibilidade de ensino flexível para os alunos, em que o ambiente de aprendizagem é capaz de adaptar os seus recursos apresentados de modo a alcançar uma aprendizagem mais efetiva, de acordo com as necessidades do aluno [Gomes et al. 2013]. Ferramentas na área da Inteligência Artificial, tais como ontologias e agentes de software podem atuar integrados nestes AVAs, sendo responsáveis por esta camada de inteligência e fazendo uso de um modelo do aluno [Viccari e Giraffa 2003]. Neste artigo, o termo "adaptação de conteúdo em AVAs" é definido como a ação de adaptar os recursos (e. g., atividades passadas, entrega de objetos de aprendizagem, mudanças na interface e auxílio personalizado) em AVAs de acordo com as características do estudante. O modelo do aluno pode ser obtido através de seu perfil. Este modelo é um registro das ações dos alunos, bem como a informação útil a respeito do aluno no ambiente de aprendizagem. Como o perfil do aluno é constituído por dados brutos dos alunos no sistema [Fröschl 2005], o modelo do aluno é composto de informações consideradas mais importantes do perfil do aluno que serão úteis para os processos de adaptação e personalização em AVAs. Por exemplo, estes dados podem ser informações pessoais, preferências e desempenho em atividades. Instituições e organizações internacionais têm estabelecido a padronização de modelo de aluno utilizado em AVAs, sendo que o padrão mais conhecido atualmente é o Instructional Management Systems Learning Information Package (IMS LIP) [LIP 2014].

No entanto, apesar da crescente utilização de ambientes educacionais, eles geralmente oferecem recursos de aprendizagem da mesma forma para todos os alunos, resultando que a aprendizagem pode não se tornar efetiva para todos, devido às diversas características cognitivas que cada aluno possui e devido às diferentes características de equipamentos que são utilizados pelos alunos para acessar o AVA. Isto, portanto, cria dificuldades de aprendizagem para alguns alunos ou até mesmo a falta de interesse por parte dos alunos no uso do ambiente de aprendizagem. Além disso, apesar de existirem várias técnicas de adaptação de recursos de aprendizagem para alunos por meio de sistemas sensíveis ao contexto [Saccol et al. 2009], apoio a formação de grupos [Hummel, Kopecny e Kotsis 2003] e personalização com agentes [Doherty et al. 2006], nem todos contemplaram as diversas características que o aluno possui. Além disso, muitos apresentaram importantes inovações tecnológicas, mas não foram apoiadas por uma teoria educacional que pudesse promover o aprendizado efetivo do estudante.

Como um cenário de exemplo, em uma Instuição de Ensino Superior (IES) temos em especial na disciplina de Cálculo Numérico, componente curricular de cursos de Ciências Exatas, atividades para resolver problemas numéricos (e.g., sistemas lineares, interpolação e regressão linear). Estes problemas numéricos podem ser resolvidos com a ajuda de um computador, permitindo que o aluno desenvolva scripts em linguagens de programação para realizar seus cálculos, que manualmente é mais complicado fazer. No entanto, muitos estudantes de Cálculo Numérico carecem de um conhecimento prévio básico de programação de computadores, faltando esta habilidade para o bom desempenho dos alunos no curso. Um estudante pode ter um bom conhecimento em cálculos matemáticos para resolver uma atividade, mas pode não ser capaz de transcrever a lógica para realizar estes cálculos em uma linguagem de programação.

Tendo como objetivo a solução deste problema, neste artigo propõe-se um modelo de aluno multi-característico que abrange quatro itens para descrever o aluno: (a) desempenho em atividades e frequência no ambiente educacional; (b) os estilos de aprendizagem; (c) o equipamento que o aluno usa, por exemplo, um PC ou um dispositivo móvel e (d) suas competências e habilidades. O padrão LIP foi estendido para integrar todas essas quatro características. Portanto, a ideia central deste trabalho é focada no desenvolvimento de um modelo de aluno a partir desta extensão do padrão LIP, consistindo de uma ontologia que descreve esse modelo que é usada por um sistema multiagente. Este, por sua vez, testa a eficácia do modelo de aluno aplicado, com o objetivo de proporcionar adaptações 
construtivistas no AVA. Com a integração das tecnologias de agentes de software e da ontologia de modelo de aluno, o AVA estará apto a adaptar recursos de aprendizagem de acordo com as necessidades dos estudantes. O modelo de aluno também é apresentado ao estudante, sendo um Modelo de Aluno Aberto (MAA). Usar MAA refere-se a tornar o modelo de aluno explícito ao estudante, de modo a fornecer um recurso adicional através do autoconhecimento e possível autorregulação do processo de aprendizagem, com o objetivo de melhorar a aprendizagem e autonomia do aluno [Bull, Quigley e Mabbott 2006]. Portanto, neste trabalho foi escolhido usar MAA.

Assim, além de proporcionar o MAA, outros recursos de ensino podem ser dispostos com maior facilidade pela adaptação do AVA, tais como: formação de grupos de alunos; fornecimento de objetos de aprendizagem de acordo com as necessidades dos alunos; recomendação de estudantes para apoiar os outros com dúvidas; atividades construtivistas e colaborativas por meio de arquiteuras pedagógicas [Castro e Menezes 2011]; correção colaborativa (revisão em pares entre os colegas, fornecendo feedbacks); e disposição de desafios abertos através do diálogo contínuo com o aluno. Acredita-se que desta maneira o AVA terá um curso com melhor sensibilidade ao contexto e será capaz de reagir de acordo com as interações dos alunos.

Além desta Introdução, o artigo está estruturado da seguinte forma: a Seção 2 discute o uso de práticas construtivistas e modelo de aluno em AVAs. A Seção 3 apresenta uma visão geral do sistema proposto e sua arquitetura, descrevendo também o modelo de aluno. A Seção 4 apresenta testes realizados de adaptação de recursos em uma disciplina de Cálculo Numérico em um AVA. A Seção 5 apresenta as conclusões e trabalhos futuros.

\section{Uso de Práticas Construtivistas e Modelo de Aluno em AVAs: Trabalhos Relacionados}

O uso de práticas construtivistas relacionadas com o modelo de aluno em AVAs tem sido uma alternativa utilizada para apoiar a tomada de decisões nos processos de ensino e aprendizagem mediados pela tecnologia. Como forma de superar o sentimento geral de isolamento e consequente alta evasão de alunos em AVAs, o trabalho de [Oliveira e Tedesco 2009] apresenta os resultados de um experimento com o modelo chamado $i$ Collaboration que promove a colaboração entre os usuários em AVA. O i-Collaboration baseia-se na utilização de agentes companheiros virtuais de aprendizagem. Por sua vez, o Sistema Inteligente de Desafios Abertos (IOCS) [Bastos Filho et al. 2006] consiste em um sistema multiagente que realiza a mediação entre o sistema e o usuário. O sistema tem a proposta de trabalhar a aprendizagem através da aplicação de desafios que consistem em problemas lógico-matemáticos de concepção aberta, preocupando-se em reconhecer como os alunos respondem as atividades propostas.

O Sistema Tutor Inteligente Conversacional Oscar (CITS) em [Latham, Crockett e McLean 2013] é um sofisticado sistema que utiliza uma interface de linguagem natural para permitir aos alunos construir seu próprio conhecimento através de discussões. O Oscar CITS imita um tutor humano, detectando e adaptando-se de forma dinâmica em função dos estilos de aprendizagem dos alunos, durante a conversação com o aluno. Usando tecnologias móveis, o paradigma educacional considerado no projeto COMTEXT [Saccol et al. 2009] é interacionista-construtivista. As ferramentas dentro do ambiente de aprendizagem COMTEXT têm como objetivo apoiar o desenvolvimento de competências, promovendo a interação entre a comunidade de alunos, com foco no compartilhamento de conhecimento e o desenvolvimento de habilidades e atitudes.

Utilizando a abordagem de modelo de aluno, o trabalho apresentado em [Graf e Kinshuk 2009] introduz um mecanismo de adaptação que é baseado em uma abordagem 
avançada de modelagem de aluno que identifica os estilos de aprendizagem. Com base nos estilos de aprendizagem identificados, o mecanismo adaptativo compõe cursos que correspondem a esses estilos, com o objetivo de tornar a aprendizagem mais fácil para os alunos. São usados os estilos de aprendizagem de Felder-Silverman [Felder e Silverman 1988]. Lidando com MAA, [Ahmad 2013] usa um Modelo de Aluno Aberto simples, apresentando o interesse dos alunos em ver seu modelo de aprendiz e considera o potencial de MAAs como uma abordagem para incentivar o estudo auto-dirigido ao longo da vida entre os estudantes em Instituições de Ensino Superior.

Assim, podemos ver que existem vários estudos na literatura que lidam com características construtivistas e modelo de aluno aplicado aos AVAs. Estes trabalhos nos ajudam a entender como tal abordagem está sendo aplicada na literatura. No entanto, observou-se com estes trabalhos descritos acima que, embora existam muitos trabalhos sobre adaptação de AVAs usando Construtivismo e modelos de aluno, muitos destes estudos utilizam características específicas dos alunos de forma separada. Em outras palavras, alguns trabalhos lidam apenas com estilos de aprendizagem, outros com competências dos alunos; outros, apenas dependendo do equipamento que o aluno utiliza. Assim, o modelo de aluno não é capaz de informar ao AVA do perfil global do aluno e sua situação atual com maior precisão no ambiente de aprendizagem. Consequentemente, fica difícil para o AVA tomar decisões corretas na tentativa de auxiliar o aluno em suas necessidades reais. Este trabalho difere ao considerar várias características dos estudantes ao mesmo tempo, variando de acordo com a interação dos alunos com o AVA e também ao apresentar o modelo de aluno aberto para o mesmo, a fim de obter um diagnóstico mais preciso da situação dos alunos no ambiente educacional e estimulando-os a aprender cada vez mais, além de proporcionar adaptação de recursos, em função de seu modelo de aluno. A próxima seção descreve a proposta com mais detalhes.

\section{Métodos Empregados}

\subsection{Arquitetura do Sistema}

A arquitetura geral do sistema é mostrada na Figura 1. O processo de adaptação começa quando o professor prepara atividades, registra estudantes e acessa o AVA. O estudante, por sua vez, interage com o AVA, seja por registro dos seus dados pessoais, postando algo no fórum ou resolvendo atividades propostas no ambiente. Para este trabalho, utilizamos o AVA Moodle [Moodle 2014].

Além disso, no AVA foram criados três questionários para os alunos responderem no início do curso, com o objetivo de obter o modelo de aluno inicial, porque esta é a primeira informação que compõe a base de conhecimento sobre os alunos no banco de dados. Os questionários são: 1) Índice de Estilos de Aprendizagem (Index of Learning Styles), buscando conhecer os estilos de aprendizagem de Felder-Silvernam [Felder e Silverman 1988] dos estudantes; 2) Questionário de Estilos de Aprendizagem de HoneyAlonso [Alonso e Gallego 2014]. Optou-se por escolher estes dois questionários visando ter uma informação mais precisa a respeito dos alunos quanto aos seus estilos de aprendizagem, obtidos pela combinação dos dois questionários; 3) Questionário sobre programação de computadores, onde os alunos irão responder a perguntas sobre o seu nível de conhecimento em conceitos de programação referentes a disciplina de Cálculo Numérico (estruturas condicionais; repetição; estruturas de dados; nível de experiência no uso de uma linguagem de programação, escolhido para esta disciplina o Scilab [Scilab 2014], e a experiência de usar o computador em Cálculo Numérico). Os links para acessar estes questionários e para acessar o Modelo de Aluno Aberto ficam disponíveis no AVA. 
Os agentes foram desenvolvidos nos frameworks JADE [JADE 2014] e JADEX [JADEX 2014]. Os agentes JADE manipulam os dados dos alunos e seus modelos de aluno no banco de dados do AVA, executando atualizações. O modelo de aluno é descrito por sua ontologia, que contém informações de estilos de aprendizagem, equipamento que o aluno pode estar utilizando, as competências do aluno, as atividades que o aluno faz e a frequência no AVA. A ontologia também é útil para a manipulação correta de troca de mensagens entre os agentes. Por sua vez, os agentes JADEX, a partir dos dados obtidos do modelo de aluno e usando o modelo Beliefs-Desires-Intentions (BDI) [Georgeff et al. 1999], irá selecionar estratégias construtivistas contidas no repositório de estratégias, como por exemplo, arquiteturas pedagógicas (que ficam no banco de dados) que serão utilizadas pelo AVA de modo que os recursos do ambiente possam ser adaptados para os alunos. Finalmente, o aluno acessa o AVA adaptado e o seu MAA.

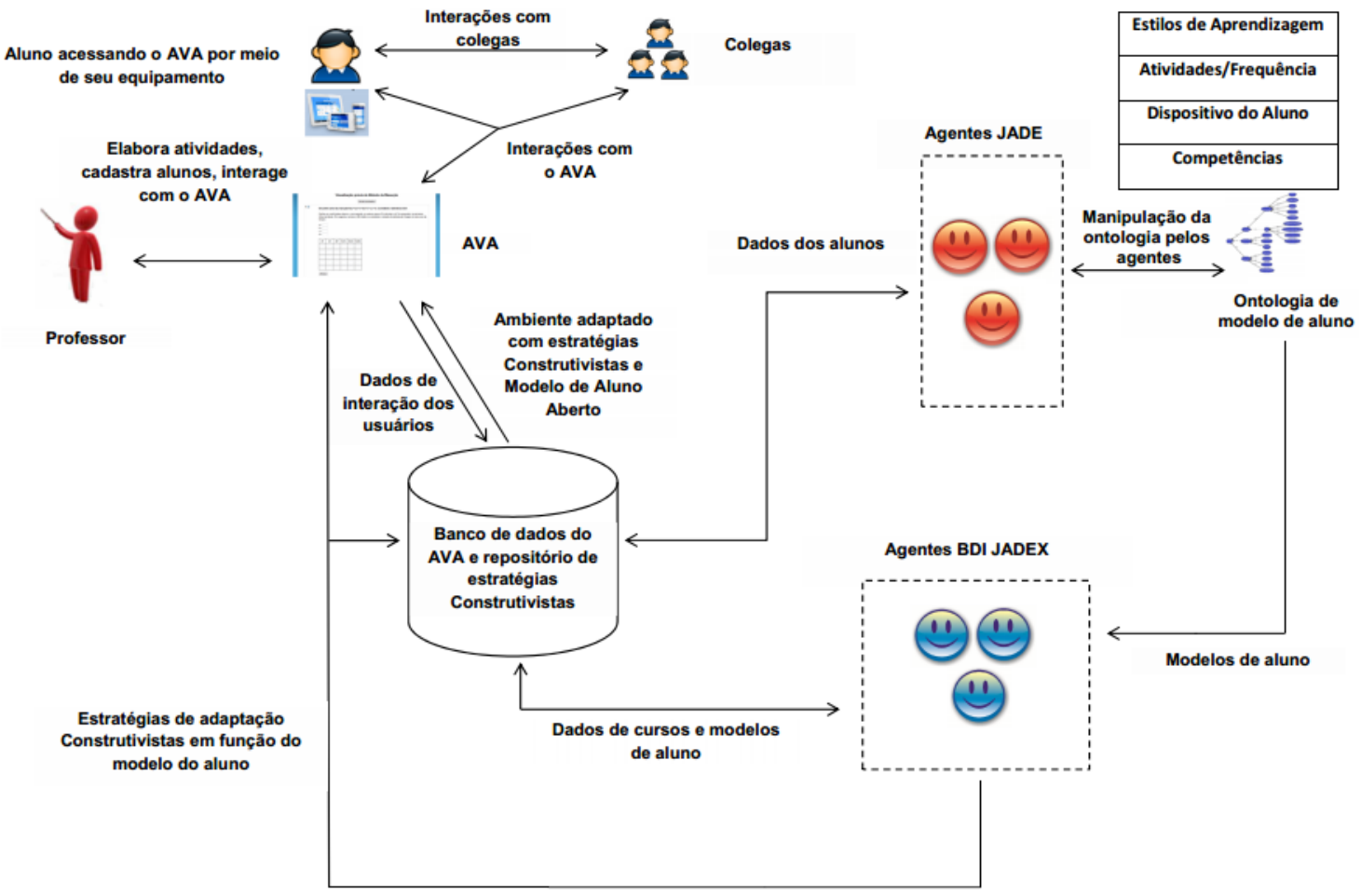

Figura 1. Arquitetura do Sistema.

\subsection{A Ontologia de Modelo de Aluno Aberto}

Outra importante etapa neste trabalho foi construir a ontologia de domínio que representa o modelo de aluno. Para o modelo de aluno foi utilizado o padrão IMS LIP [LIP 2014], que é suportado por meio de exemplos do modelo, além de ser um padrão largamente usado hoje em dia e que permite extensões. Originalmente, o LIP tem 11 categorias de dados do estudante: Identification, Accessibility, Goal, QCL, Activity, Competency, Interest, Affiliation, Transcription, Security key e Relationship. No entanto, mesmo o LIP tendo estas categorias, os dados que descrevem as habilidades específicas dos alunos, seus estilos de aprendizagem e os dados sobre os equipamentos utilizados não são especificados no LIP, e para determinadas disciplinas ou cursos específicos, pode não descrever corretamente características importantes do aluno. Assim, mesmo que tenham sido usadas cinco categorias do LIP (Activity, Competency, Identification, Accessibility e Interest), este padrão foi estendido para as necessidades deste trabalho, enfatizando as habilidades do 
aluno, o equipamento que ele usa (desktop, smartphone, tablet), as atividades respondidas, a frequência dos acessos no AVA e os estilos de aprendizagem do aluno.

$\mathrm{Na}$ categoria Activity são informadas as atividades propostas pelo professor. A ontologia de modelo de aluno na classe Competency descreve o nível de habilidade de cada aluno, o que é útil para identificar os alunos e seus níveis de competências e habilidades em uma disciplina ou curso. Em nossa proposta, cada competência do aluno é composta por um conjunto de habilidades específicas. Por exemplo, na disciplina de Cálculo Numérico, a competência "Encontrar raízes de funções pelo método da bisseção" consiste nas seguintes habilidades: "Manuseio da máquina de calcular", "Compreensão de funções (continuidade, Teorema do Valor Intermediário, gráficos de plotagem, convergência e divergência)" e "Compreender as operações do método da bisseção (escolha do intervalo inicial, a fórmula e os critérios para a escolha do novo intervalo)" [Burden e Faires 2010]. Os agentes podem fazer avaliações frequentes das atividades dos alunos, sendo uma avaliação formativa, obtendo de seu desempenho e atualizando os níveis de habilidades dos alunos. Outras categorias utilizadas são Identification (para os dados pessoais do aluno), Accessibility (dados de acessibilidade do usuário, credenciais no AVA) e Interest (contendo os interesses dos estudantes). As outras seis categorias restantes do LIP não foram usadas porque elas não fazem parte do escopo deste trabalho, ou já são substituídas pelas categorias que foram utilizadas, evitando qualquer conflito de informações com dados semelhantes. No entanto, o padrão LIP foi estendido em três outras categorias (ou classes): CCPP_Device, Learning_Styles e Frequency. A categoria CCPP_Device descreve os dispositivos utilizados pelos alunos. Assim como há padrões para o modelo do aluno, permitindo adaptação do conteúdo, existem normas para a caracterização de dispositivos móveis, com as suas características como tamanho da tela, desempenho da CPU, memória, entre outros. Um dos mais usados atualmente e usado neste trabalho é o Composite Capabilities/Preference Profile (CC/PP) [CC/PP 2014]. A caracterização de dispositivos móveis pode ser considerada como o perfil do dispositivo, que é útil para a adaptação de conteúdo para ser apresentado aos estudantes dependendo do dispositivo. Usando a categoria Frequency, esta descreve a frequência dos alunos no AVA, em que é verificado se em um período contínuo de 20 dias o aluno não acessou o AVA e também é verificado se o aluno terminou ou não suas atividades. Finalmente, a categoria Learning_Styles contém informações sobre os estilos de aprendizagem dos alunos Felder-Silvernam e HoneyAlonso.

A ontologia de modelo de aluno foi desenvolvida no editor Protégé [Protégé 2014] e tem três classes principais: Concept, cujas filhas são as subclasses $A I D$ (descrevendo os agentes que fazem parte do sistema multiagente) e Learner_LIP (contendo as categorias LIP utilizadas neste trabalho, além do CCPP_Device, Learning_Styles e Frequency); Predicate, responsável pelos fatos sobre o ambiente onde os agentes atuam; e AgentAction (contendo as ações que os agentes realizam no AVA). Todas estas três classes permitem que a ontologia seja integrada ao sistema multiagente. A Figura 2 mostra uma representação gráfica resumida da ontologia de Modelo de Aluno Aberto. Na Figura 2 é mostrada apenas a subclasse Learner_LIP de Concept, que descreve o padrão LIP estendido e apresenta os slots (propriedades ou atributos) das demais classes. Por exemplo, na classe Learning_Styles, uma propriedade é $l s \_f$ _active_reflective, indicando se o aluno tende a ser mais ativo ou reflexivo nos estilos de aprendizagem de Felder-Silvernam. O único AID apresentado na Figura 2 é o Update Profile Agent, desenvolvido no JADE, responsável por atualizar os dados do modelo de aluno a partir das interações do aluno com o AVA. As relações e regras entre as classes descrevem os facets da ontologia, representados por setas 
azuis e pretas (estas últimas indicando apenas a relação "isa", que significa "é um", de subclasse para superclasse). Como alguns exemplos de facets em setas azuis na Figura 2, o estudante faz uma atividade (doesActivity) e tem dúvidas ou erros (hasDoubts_Errors). Estes dois facets ligam as classes Identification e Activity. Além disso, o estudante tem competências e habilidades (hasSkillsCompetencies). Esse facet liga as classes Identification e Competency.

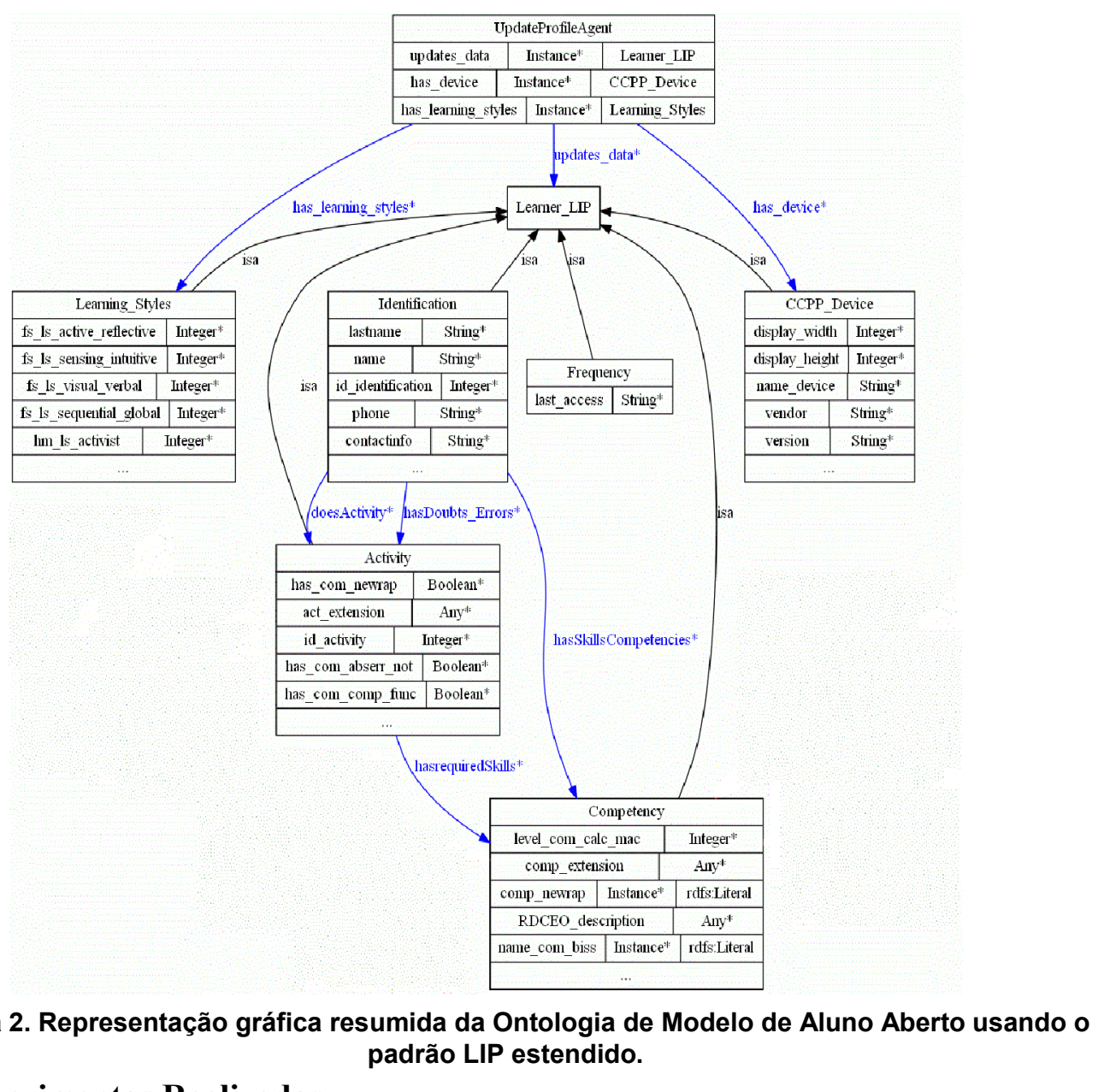

\section{Experimentos Realizados}

\subsection{Apresentando o Modelo de Aluno Aberto aos Alunos}

O aluno pode acessar seu modelo por meio de um link no AVA. É importante que o MAA seja facilmente compreendido pelo aluno. Assim, a Figura 3 mostra um exemplo de MAA apresentado aos alunos composto de três gráficos, que é o resultado das respostas dos alunos aos questionários. No primeiro gráfico são apresentados os estilos de aprendizagem de acordo com o questionário de Honey-Alonso, em porcentagens dos estilos Ativo, Reflexivo, Teórico e Pragmático. No segundo, os estilos de Felder-Silvernam (Ativo/Reflexivo, Sensorial/Intuitivo, Visual/Verbal e Sequencial/Global) são apresentados em valores ímpares que variam de -11 a +11 . Por exemplo, se um aluno tem no campo Visual/Verbal valor +7 , isso significa que ele é mais Visual. Se fosse -7 , ele seria mais Verbal. Finalmente, o terceiro gráfico mostra as habilidades do aluno em um curso específico, no nosso estudo de caso, Cálculo Numérico. Além disso, na Figura 3 é mostrado se o estudante usa ou não dispositivos móveis para acessar o AVA, e esta informação é 
dinamicamente alterada (pois um estudante pode estar usando ou não um dispositivo móvel em um determinado momento) e um link para as situações em que o aluno não concorda com o seu modelo de aluno mostrado. Nesse link, um aluno pode enviar uma mensagem para o sistema, informando que não concorda com seu modelo mostrado. Esse link é útil para detectar se há alunos que concordam ou não com seus respectivos modelos de aluno, uma vez que é possível que um estudante se julgue autossuficiente e muito capaz no curso, mas não seja considerado capaz pelas avaliações feitas pelo sistema. Com base na resposta do aluno, os agentes JADE farão uma nova atualização no modelo do aluno.

\subsection{Técnicas de Adaptação Testadas}

No AVA foram testadas duas técnicas de adaptação de recursos, usadas em uma turma composta por 33 alunos da disciplina de Cálculo Numérico na Universidade Federal do Amazonas (UFAM). Essas duas técnicas são o monitoramento e a recomendação de colegas para ajudar os alunos que tinham dúvidas em atividades.

Meu Modelo de Aluno Aberto Seu nome: aluno1

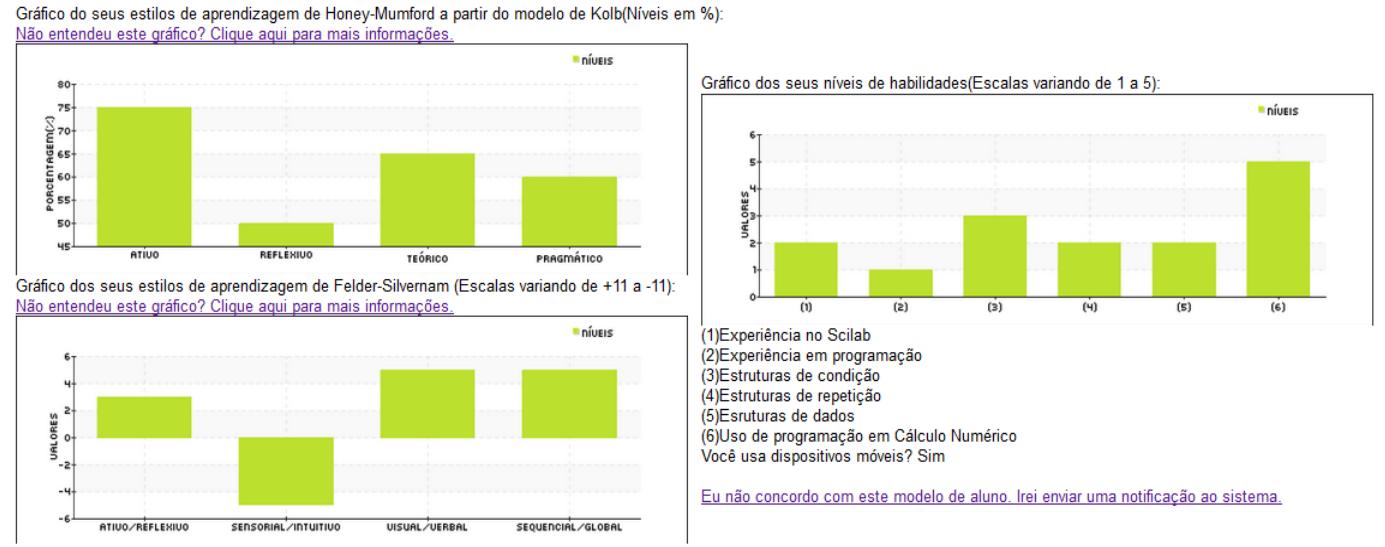

Figura 3. Apresentando o Modelo de Aluno Aberto a estudantes.

O processo ocorre da seguinte forma: os alunos acessam o AVA, executam as atividades propostas pelo professor e dúvidas ou erros podem surgir, detectado pelo monitoramento. Ao mesmo tempo em que os alunos interagem com o AVA, seus modelos de aluno são atualizados no banco de dados através do sistema multiagente, que também é responsável, juntamente com a ontologia, por procurar pelos alunos com melhores níveis de habilidades específicas, capazes de ajudar o estudante que estiver com dúvidas nas atividades. Assim, os estudantes com perfis apropriados encontrados pelo sistema multi-agente serão recomendados no AVA para o aluno que tem dúvidas ou errou as atividades. A Figura 4(a) mostra as mensagens trocadas entre dois agentes implementados no framework JADE. Um deles é o Doubt \& Error Profile Agent, responsável por encontrar estudantes com dúvidas ou erros nas atividades e que faz requisições ao Recommended Profile Agent, para que este agente selecione os estudantes que irão ajudar seus colegas com dúvidas. A lista detalhada de todos os agentes pode ser encontrada em [Bremgartner e Netto 2011, 2012].

A Figura 4(b) mostra uma notificação através do Help Tutor Agent para o aluno que não conseguiu terminar a atividade no tempo especificado pelo professor. Nesta mensagem de notificação há um link para o aluno clicar para obter uma tela de colegas recomendados pelo sistema que podem ajudá-lo. Esta tela é mostrada na Figura 4(c), numa tabela que indica o nome da atividade, os colegas recomendados para ajudar nessa atividade e os interesses destes colegas recomendados. Essas informações são úteis para incentivar a interação entre os alunos. No final destes dois testes foi observada uma melhoria de $180 \%$ de acerto dos alunos. Dos 33 alunos, apenas quatro inicialmente responderam corretamente todas as atividades propostas. 
Após a aplicação do recurso de recomendação, 12 alunos responderam corretamente as mesmas atividades. Podemos observar com estes testes que a notificação para os alunos com problemas em atividades e a sugestão de colegas para ajudar os alunos com dúvidas são técnicas construtivistas úteis para melhorar o aprendizado do aluno e que incentivam a prática de atividades colaborativas em AVAs.

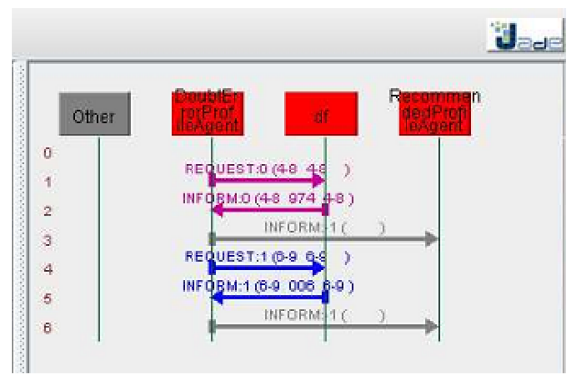

(a)

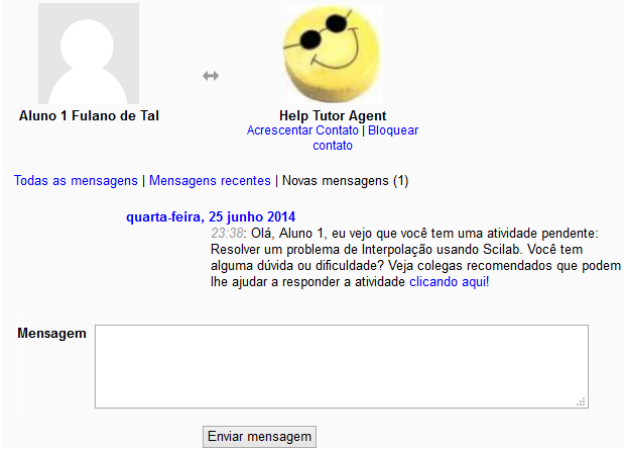

(b)

Dúvidas em atividades? Veja aqui colegas que podem ajudar!

\begin{tabular}{|lll|}
\hline Atividade & Estudantes Recomendados & Interesses \\
\hline Resolver o problema do paraquedista usando Interpolaçäo no Scilab & Aluno 2 & programaçẫo \\
Resolver o problema do paraquedista usando Interpolação no Scilab & Aluno 3 & cảlculos, operar máquinas \\
\hline
\end{tabular}

(c)

Figura 4. (a) Troca de mensagens entre agentes de recomendação no JADE. (b) Mensagem enviada pelo Help Tutor Agent ao aluno. (c) Estudantes recomendados.

\section{Conclusões e Trabalhos Futuros}

Este artigo mostrou uma proposta de adaptação de recursos em AVAs baseada na teoria construtivista da aprendizagem. Foram desenvolvidos um sistema multiagente e uma ontologia de Modelo de Aluno Aberto no padrão IMS LIP capazes de selecionar técnicas de adaptação no AVA Moodle. Esta estratégia permite uma maior personalização dos recursos apresentados em função das características dos alunos. O processo de adaptação descrito na arquitetura do sistema aumenta a interação entre os alunos de um mesmo curso ou disciplina e a interação dos alunos com o AVA, aumentando assim o aprendizado efetivo e maiores acertos nas tarefas executadas. Os testes realizados mostraram que a recomendação de alunos para ajudar os outros com dúvidas através da aprendizagem colaborativa é uma solução que pode ajudar a construir o conhecimento do aluno de forma útil e eficaz para o problema da falta de auxílio para os alunos com dúvidas em AVAs. A abordagem de sistema multiagente, juntamente com o MAA, pode ser aplicada a outros AVAs, uma vez que os agentes e o ambiente educacional podem compartilhar a mesma base de dados. Como trabalhos futuros, a ideia é testar o sistema para adaptação de conteúdo em dispositivos móveis, visto que a ontologia já descreve os recursos do dispositivo móvel no padrão CC/PP. Além disso, enquanto a ontologia e o sistema multiagente proporcionam a adaptação de recursos, o próximo passo é também recomendar diversos tipos de atividades e propostas de trabalho construtivistas com basadas em arquiteturas pedagógicas, como júri simulado, diário virtual e debate de teses.

\section{Referências}

Ahmad, N. (2013) "Self-Directed Learning: Student's Interest in Viewing the Learner Model”. Proceeding of 3rd International Conference on Research and Innovation in Information Systems (ICRIIS), pp. 493-498, Malásia.

Alonso, C. e Gallego, D. (2014) Questionário Honey-Alonso de Estilos de Aprendizagem, http://www.estilosdeaprendizaje.es/chaea/chaeagrafp2.htm. 
Bastos Filho, O. C., Axt, M., Labidi, S. e Machado, C. T. (2006) "Sistema Inteligente de Desafios Abertos - IOCS: Uma proposta de adaptação dos padrões do Método Clínico Piagetiano em Plataforma Multiagentes”. CINTED-UFRGS - Novas Tecnologias na Educação, V. 4 Nº 1, Julho.

Bremgartner, V. e Netto, J. F. M. (2011) “Auxílio Personalizado a Estudantes em Ambientes Virtuais de Aprendizagem Utilizando Agentes e Competências". In: XXII Simpósio Brasileiro de Informática na Educação - SBIE2011, Aracaju, SE. Anais do XXII SBIE - XVII WIE. Porto Alegre: SBC Sociedade Brasileira de Computação, p. 40-49.

Bremgartner, V e Netto, J. F. M. (2012) "Improving Collaborative Learning by Personalization in Virtual Learning Environments Using Agents and Competency-Based Ontology". Proceedings of IEEE Frontiers in Education Conference (FIE 2012), Seattle, EUA.

Bull, S., Quigley, S. e Mabbott, A. (2006) “Computer-based Formative Assessment to Promote Reflection and Learner Autonomy”. Engineering Education, vol. 1, no. 1, pp. 8-18.

Burden, R. L. e Faires, J. D. (2010) Numerical Analysis. Brooks/Cole, Cengage Learning, $9^{\text {th }}$ Ed., Boston, EUA.

Castro, A. N. e Menezes, C. S. (2011) “Aprendizagem Colaborativa com Suporte Computacional”. In: Pimentel, M. e Fuks, H. Sistemas Colaborativos. Rio de Janeiro: Campus. ISBN: 978-85-352-4669-8.

CC/PP. (2014) Composite Capabilities/Preference Profiles, www.w3.org/Mobile/CCPP/

Doherty, B. C. et al. (2006) "Entre-pass: Personalising u-Learning with Intelligent Agents". Proceedings of Fourth IEEE International Workshop on Wireless, Mobile and Ubiquitous Technology in Education (WMUTE’06), Atenas, Grécia.

Felder, R. M. e Silverman, L. K. (1988). "Learning and teaching styles in engineering education". Engineering Education, 78, pp. 674-681.

Fröschl, C. (2005) "User Modeling and User Profiling in Adaptive E-learning Systems". Tese de Mestrado. Institute for Information Systems and Computer Media, Graz University of Technology, Graz, Áustria.

Georgeff, M., Pell, B., Pollack, M., Tambe, M. e Wooldridge, M. (1999) “The Belief-Desire-Intention Model of Agency". In: Intelligent Agents V, LNAI 1555, Springer, pp. 1-10.

Gomes, E.H., Pimentel, E. P., Omar, N., Marietto, M. G. B. (2013) "Personalização do E-Learning Baseado no Nível de Aquisição de Conhecimentos do Aprendiz". Anais do XXIV Simpósio Brasileiro de Informática na Educação (SBIE), Campinas, SP.

Graf, S. e Kinshuk. (2009) "Advanced Adaptivity in Learning Management Systems by Considering Learning Sytles”. Proceedings of IEEE/WIC/ACM International Conference on Web Inteligence and Intelligent Agent Technology.

Hummel, K. A., Kopecny, R. e Kotsis, G. (2003) "Peer Profile Driven Group Support for Mobile Learning Teams". Proceedings of Computers and Advanced Technology in Education, Rhodes, Grécia.

JADE. (2014) Java Agent DEvelopment Framework, www.jade.tilab.com/

JADEX. (2014) JADEX Active Components, www.activecomponents.org/bin/view/About/Features

Latham, A., Crockett, K. e McLean, D. (2013) "An adaptation algorithm for an intelligent natural language tutoring system”. Computers \& Education 71, Elsevier, pp. 97-110

LIP. (2014) Learner Information Package Specification 1.0.1, www.imsglobal.org/profiles/lipinfo01.html\#5.1/

Moodle (2014) A Free, Open Source Course Management System for Online Learning, www.moodle.org/

Oliveira, E. A. e Tedesco, P. (2009) "i-Collaboration in Practice: Results from our Investigation within the Cleverpal Environment". Proceedings of IEEE International Conference on Intelligent Computing and Intelligent Systems (ICIS), pp. 347-351.

Protégé. (2014) The Ontology Editor, www.protege.stanford.edu/

Saccol, A. Z., Kich, M., Schlemmer, E., Reinhard, N., Barbosa, J. L. V. e Hahn, R. (2009) “A Framework for the Design of Ubiquitous Learning Applications". In 42nd Hawaii International Conference on System Sciences Proceedings.

Scilab. (2014) Open source software for numerical computation, www.scilab.org/

Viccari, R. M. e Giraffa, L. M. M. (2003) “Fundamentos dos Sistemas Tutores Inteligentes”. In: Barone, D. (Org). Sociedades artificiais: a nova fronteira da inteligência das máquinas. Porto Alegre: Bookman. ISBN: 85-363-0124-4. 